\title{
The Avoidance of the Little Sibling of the Big Rip Abrupt Event by a Quantum Approach
}

\author{
Imanol Albarran 1,2,*iD, Mariam Bouhmadi-López ${ }^{3,4}$ Franciso Cabral $^{5}$ and \\ Prado Martín-Moruno 6,7 \\ 1 Departamento de Física, Universidade da Beira Interior, Rua Marquês D’Ávila e Bolama, \\ 6200-001 Covilhã, Portugal \\ 2 Centro de Matemática e Aplicações, Universidade da Beira Interior, Rua Marquês D’Ávila e Bolama, \\ 6200-001 Covilhã, Portugal \\ 3 Department of Theoretical Physics, University of the Basque Country, UPV/EHU, P.O. Box 644, \\ 48080 Bilbao, Spain; mariam.bouhmadi@ehu.eus \\ 4 Ikerbasque, Basque Foundation for Science, 48011 Bilbao, Spain \\ 5 Instituto de Astrofísica e Ciências do Espaço, Faculdade de Ciências da Universidade de Lisboa, Edifício C8, \\ Campo Grande, 1749-016 Lisbon, Portugal; fc30808@alunos.fc.ul.pt \\ 6 Departamento de Física Teórica, Universidad Complutense de Madrid, E-28040 Madrid, Spain; \\ pradomm@ucm.es \\ 7 UPARCOS, Universidad Complutense de Madrid, E-28040 Madrid, Spain \\ * Correspondence: imanolalbarran@gmail.com or albarran.payo@ubi.pt
}

Received: 29 November 2017 ; Accepted: 22 January 2018 ; Published: 6 February 2018

\begin{abstract}
We address the quantisation of a model that induces the Little Sibling of the Big Rip (LSBR) abrupt event, where the dark energy content is described by means of a phantom-like fluid or a phantom scalar field. The quantisation is done in the framework of the Wheeler-DeWitt (WDW) equation and imposing the DeWitt boundary condition; i.e., the wave function vanishes close to the abrupt event. We analyse the WDW equation within two descriptions: First, when the dark energy content is described with a perfect fluid. This leaves the problem with the scale factor as the single degree of freedom. Second, when the dark energy content is described with a phantom scalar field in such a way that an additional degree of freedom is incorporated. Here, we have applied the Born-Oppenheimer (BO) approximation in order to simplify the WDW equation. In all cases, the obtained wave function vanishes when the LSBR takes place, thus fulfilling the DeWitt boundary condition.
\end{abstract}

Keywords: dark energy; cosmological singularities; quantum cosmology

\section{Introduction}

It can be said that the science of Cosmology was born with Einstein's General Relativity (GR) at the beginning of the 20th century. This beautiful theory set the foundations to describe the Universe as a whole, relating the movement of matter with the geometry as if it was a graceful dance. The evolution of the Universe is ruled by Einstein field equations, where the components that fill the Universe determine the expansion rate at different cosmological eras. For a long time, it was assumed that the Universe was mainly composed by radiation and matter. This led to presume a Universe that was born at the so-called Big Bang singularity, evolving with a negative acceleration towards a final doomsday known as Big Crunch (for closed space hypersurfaces). However, the discovery of the current accelerating expansion of the Universe in the late 1990s changed the idea of such doomsday. That acceleration was empirically supported by SNeIa observations [1,2] and later on by independent cosmological observations $[3,4]$. The new discovery brought new problems and new enigmas, which are still today 
considered as one of the major open problems in cosmology. The raised issues regarding the final doomsday, if any, and what kind of mechanism could induce such acceleration, have yet to be resolved.

Several works try to explain the current cosmological speed up invoking an exotic component in the Universe known as Dark Energy (DE) [5-7] where the $\Lambda$ CDM model is the widely accepted paradigm given its remarkable success on fitting the observational data. However, this model brings certain theoretical problems such as the coincidence problem and the cosmological constant problem. The $\Lambda$ CDM model is distinguished by having an Equation of State (EoS) parameter (which is simply the ratio between the pressure and energy density), $w_{\mathrm{d}}=-1$, where the asymptotic evolution lead to a de Sitter Universe. Although the $\Lambda$ CDM model gives the best observational fit, there is no reason to exclude other models that could describe the Universe in a suitable way. In particular, phantom models are viable (see Ref. [3])

Among the huge amount of DE models, many of them predict future singularities or abrupt events. A cosmological singularity occurs when a cosmological variable diverges. We name as singularity, if it takes place at a finite cosmic time, or abrupt event, if it happens at an infinite cosmic time.

The field of cosmological singularities was first studied in $[8,9]$, where the authors contemplated the future singularity of the Big Crunch. However, the discovery of the recent speed up has yield to new kind of future singularities such as Sudden Singularity [10-14], Big Freeze [15-18] and Type IV [13,14,19-22]. Moreover, the observations do not exclude the models known as phantom, where the null energy condition, i.e., $0 \leq p+\rho$, is violated. The singularities and abrupt events genuinely related to such kind of energy condition are ${ }^{1}$ : Big Rip (BR) [23-30] , Little Rip (LR) [31-36] and Little Sibling of the Big Rip (LSBR) [37,38].

Among the phantom induced cosmological events, we have focused on the LSBR. This abrupt event was first analysed in [37]. There, the authors suggest a particular EoS for dark energy in a homogeneous and isotropic Universe. After solving the Friedmann and Raychaudhuri equations, the asymptotic evolution leads to a Universe where the scale factor and the Hubble rate diverge at infinite cosmic time while the first cosmic time derivative of the Hubble rate remains constant.

Close to the abrupt event, for example, the energy density reaches very large values and quantum effects are expected to become important. Therefore, it is necessary to implement a quantum analysis. The main motivation of this work consists on avoiding the singularities that arise in the classical theory of GR through a quantum approach. The hope is that a consistent theory of quantum gravity, should in principle, avoid the divergences of the classical theory of gravity. Unfortunately, we do not yet have a complete theory of quantum gravity, although some recent approaches could be potentially successful in quantising gravity (see, for example, Ref. [39]). In the absence of such foundation, we have proceed with the WDW approach. This quantisation method is rooted on the framework of quantum geometrodynamics where a canonical quantisation of gravity is performed [40,41]. Avoidance consists on the compliance of the DeWitt boundary condition, according to which the wave function should vanish on those regions where the system experiences the divergence of the cosmological variables [42].

The paper is outlined as follows: In Section 2, we present a review of the phantom model that induces the LSBR abrupt event, where the dark energy content is described first with a phantom-like fluid, and, then, with a phantom scalar field. In Section 3, we introduce the WDW equation and present the results obtained when describing the matter content with a perfect fluid, where we address two types of quantisation procedures. Then, we analyse the WDW equation when describing the matter content with a scalar field, where a second degree of freedom is incorporated. Finally, in Section 4, we disclose the main conclusions.

1 The Sudden Singularity, Big Freeze and Type IV singularities can be induced in a phantom or non-phantom scenario. On the contrary, the Big Rip singularity and the abrupt events of Little Rip and Little Sibling of the Big Rip, if and only if phantom matter is present. 


\section{The Model and the LSBR Induced Abrupt Event}

In this section, we present the model that induces the future abrupt event known as LSBR. We first address the model by a perfect fluid description and then we proceed to map such a fluid to a scalar field. We first start assuming an isotropic and homogeneous Universe, whose geometry is given by a Friedmann-Lemaître-Robertson-Walker (FLRW) space-time metric

$$
d s^{2}=-N d t^{2}+a^{2}(t)\left[\frac{d r^{2}}{1-k r^{2}}+r^{2} d \theta^{2}+r^{2} \sin ^{2} \theta d \varphi^{2}\right],
$$

where $N$ is the lapse function, $a(t)$ is the scale factor and $k$ is the spatial curvature, which can take the values $k=-1,0,1$, for open, flat and closed Universes, respectively. From now on, we restrict to a spatially flat Universe, $k=0$, for simplicity and in agreement with the current observations. For such a Universe, the Friedmann and Raychaudhuri equations read

$$
\begin{aligned}
& H^{2}=\frac{8 \pi G}{3} \rho, \\
& \dot{H}=-4 \pi G(p+\rho),
\end{aligned}
$$

where $H$ is the Hubble rate, and the dot stands for derivatives with respect to cosmic time. We have set $N=1$. On the other hand, $\rho$ and $p$ are, respectively, the total energy density and pressure, given by the sum over partial contributions of all the components

$$
\rho=\rho_{\mathrm{r}}+\rho_{\mathrm{m}}+\rho_{\mathrm{d}}, \quad p=p_{\mathrm{r}}+p_{\mathrm{m}}+p_{\mathrm{d}},
$$

where the subindices $\mathrm{r}, \mathrm{m}$ and $\mathrm{d}$ denote the components of radiation, matter and dark energy, respectively. We disregard the contribution of radiation, given its negligible contribution to the total energy budget of the Universe at present. Notice that we are interested in the very late-time evolution, where the dark energy completely dominates the Universe. Moreover, at present time, the fractional energy densities of matter and dark energy are, respectively, $\Omega_{\mathrm{m} 0} \sim 0.306$ and $\Omega_{\mathrm{d} 0} \sim 0.694$ as observed by Planck mission [3,4], so for practical purposes, we can disregard the contribution of matter in the future as well. Therefore, we can approximate the total energy density and pressure to the partial contribution given by dark energy, $\rho \approx \rho_{\mathrm{d}}$ and $p \approx p_{\mathrm{d}}$. The EoS that characterises the dark energy model reads [37]

$$
p_{\mathrm{d}}=-\rho_{\mathrm{d}}-\frac{\mathcal{A}}{3},
$$

where $\mathcal{A}$ is a positive parameter that describes the model. Notice that a negative value of the constant $\mathcal{A}$ turns the model "standard" in the sense that the null energy condition is satisfied, $0 \leq \rho+p$. Contrarily, a positive value induces violations of such condition and we label it as "phantom". Just replacing the previous equation into the Raychaudhuri Equation (3), it is found that the cosmic time derivative of the Hubble rate is constant [37]

$$
\dot{H}=\frac{4 \pi G}{3} \mathcal{A} \text {. }
$$

On the other hand, we have considered that the different components are conserved separately, therefore by replacing Equation (5) in the conservation equation, $\dot{\rho}_{\mathrm{d}}=-3 H\left(\rho_{\mathrm{d}}+p_{\mathrm{d}}\right)$, we find the solution

$$
\rho_{\mathrm{d}}(a)=\rho_{\mathrm{d} 0}+\mathcal{A} \ln (a),
$$

where $\rho_{\mathrm{d} 0}$ is the current dark energy density while the scale factor at present time is settled to be $a_{0}=1$. Finally, this expression is used to solve the Friedmann equation in order to get the evolution of the scale factor in terms of cosmic time [37] 


$$
a(t) \sim \exp \left[\frac{H_{0}^{2} \Omega_{\mathcal{A}}}{4}\left(t-t_{0}\right)^{2} \pm \sqrt{\Omega_{\mathrm{d} 0}} H_{0}\left(t-t_{0}\right)\right]
$$

where $t_{0}$ denotes the present cosmic time and we have defined the new parameter ${ }^{2} \Omega_{\mathcal{A}} \equiv 8 \pi G \mathcal{A} / 3 H_{0}^{2}$. As can be seen, in the asymptotic evolution, $t \rightarrow \infty$, the scale factor and the Hubble rate blow up while the first cosmic time derivative of the Hubble rate is constant. Although the divergence of the cosmological variables occurs at an infinite cosmic time, all the bound structures will be eventually ripped apart at a finite cosmic time [37]. This abrupt event can be interpreted as the "weak" version of a Big Rip (BR) singularity shifted to infinite cosmic time, hence the name of the "Little Sibling of the Big Rip". In a BR, the Hubble rate and its cosmic time derivatives diverges.

The effective dynamics of a phantom-like fluid can be described as well by means of a phantom scalar field. This representation includes an additional degree of freedom, the scalar field $\phi$, whose energy density and pressure reads

$$
\rho=-\frac{\dot{\phi}^{2}}{2}+V(\phi), p=-\frac{\dot{\phi}^{2}}{2}-V(\phi) .
$$

Combining the latter two equations and using the equation of state (Equation (5)), we can split the scalar field and potential as

$$
\begin{aligned}
\dot{\phi} & = \pm \sqrt{\frac{\mathcal{A}}{3}}, \\
V(\rho) & =\rho+\frac{\mathcal{A}}{6} .
\end{aligned}
$$

We first solve Equation (10), which defines two solutions corresponding to the branches of the square root in Equation (10)

$$
\phi(a)= \pm \frac{1}{\sqrt{2 \pi G}}\left[\frac{\Omega_{\mathrm{d} 0}}{\Omega_{\mathcal{A}}}+\ln (a)\right]^{\frac{1}{2}}+\phi_{1}
$$

where we have fixed $\phi_{1}$ as an integration constant at some scale factor, $a=a_{1}$, large enough to be in a DE dominated Universe

$$
\phi_{1}=\phi\left(a_{0}\right) \mp \frac{1}{\sqrt{2 \pi G}} \sqrt{\frac{\Omega_{\mathrm{d} 0}}{\Omega_{\mathcal{A}}}} .
$$

Finally, we can prove that

$$
\begin{aligned}
\rho(\phi) & =2 \pi G \mathcal{A}\left(\phi-\phi_{1}\right)^{2}, \\
V(\phi) & =\frac{\mathcal{A}}{6}+2 \pi G \mathcal{A}\left(\phi-\phi_{1}\right)^{2} .
\end{aligned}
$$

As can be seen, the potential has a quadratic dependence on the scalar field.

\section{The Wheeler-DeWitt Equation}

The Wheeler-DeWitt (WDW) equation is the cosmological analogue of the Schrödinger equation in quantum mechanics. In this approach, the space-time metric and matter fields are regarded as the (canonical) variables describing the degrees of freedom to be quantised through the standard canonical quantisation procedure.

2 While we are not giving any observational constraint on the value of parameter $\mathcal{A}$, some estimation can be found in Ref. [37]. 
It is rooted in quantum geometrodynamics, providing a method for quantisation of General Relativity.

We will briefly introduce the Wheeler-DeWitt equation. The Einstein-Hilbert action for an isotropic and homogeneous Universe reads [40,41]

$$
S_{\mathrm{HE}}=\frac{3 \pi}{4 G} \int\left(-\frac{a \dot{a}^{2}}{N}+k N a\right) d t .
$$

This action corresponds to the metric and contributes to the total action as $S=S_{\mathrm{HE}}+S_{\mathrm{m}}$, where $S_{\mathrm{m}}$ is the action of the matter content. The latter can be described, for example, by a perfect fluid or by a scalar field. The variation of the Hamiltonian with respect to the lapse function $N$, leads to the Hamiltonian constraint, $\mathcal{H}=0$. Therefore, the WDW equation reads

$$
\hat{\mathcal{H}} \Psi=0 .
$$

The Hamiltonian now is an operator acting over the wave function $\Psi$, which is commonly referred as the wave function of the Universe. In the following subsections we find the solution to the WDW equation for the model considered in the previous section. The aim is to obtain a solution that complies with the DeWitt boundary condition, or equivalently, a wave function of the Universe which vanishes close to the LSBR abrupt event. We address the task of quantising the system adopting different points of view; First, the simplest case which consists in describing the content with a phantom fluid, where the scale factor is the single degree of freedom. Here, we have proceed with two different factor orderings and the solutions are obtained using the WKB approximation. Second, the matter content is described with a phantom scalar field, which incorporates a second degree of freedom in the problem. In this case, we use the Laplace Beltrami factor ordering and apply the $\mathrm{BO}$ approximation.

\subsection{WDW Equation with a Perfect Fluid}

We first start with the action where the matter content is described by a perfect fluid [40]

$$
S=\int\left[\frac{3 \pi}{4 G}\left(-\frac{a \dot{a}^{2}}{N}+k a N\right)-2 \pi^{2} N a^{3} \rho(a)\right] d t .
$$

Within this description, the scale factor is the single degree of freedom. Applying concepts from classical mechanics, we can first obtain the Lagrangian and then the Hamiltonian, using the definition $H=\sum \dot{q}_{i} p_{i}-L$. Both quantities can be written as [40]

$$
\begin{aligned}
L & =\frac{3 \pi}{4 G}\left(-\frac{a \dot{a}^{2}}{N}+k N a\right)-2 N \pi^{2} a^{3} \rho(a) \\
H & =N\left[-\frac{G}{3 \pi} \frac{p_{a}^{2}}{a}-\frac{3 \pi k}{4 G} a+2 \pi^{2} \rho(a) a^{3}\right] .
\end{aligned}
$$

where $p_{a}$ is the conjugate momenta of the scale factor and is defined as

$$
p_{a} \equiv \frac{\partial L}{\partial \dot{a}}=-\frac{3 \pi}{2 G} \frac{a \dot{a}}{N}
$$

Now, we should just apply the WDW equation, $\hat{\mathcal{H}} \Psi=0$. The exact form of the operators inside the Hamiltonian $\hat{\mathcal{H}}$, depends on the chosen factor ordering. In the following subsections, we address two kinds of quantisation procedures and we analyse if the DeWitt boundary condition is satisfied in both quantisation procedures. 


\subsubsection{First Factor Ordering}

This quantisation method simply consists on multiplying the full WDW equation by the scale factor, $a \hat{\mathcal{H}} \Psi=0$, and defineing the operator corresponding to the squared momenta as ${ }^{3}$

$$
p_{a}^{2} \rightarrow \hat{p}_{a}^{2}=-\hbar^{2} \frac{d^{2}}{d a^{2}}
$$

With this factor ordering, the WDW equation reads

$$
\left\{\frac{d^{2}}{d a^{2}}+\frac{6 \pi^{3}}{\hbar^{2} G} a^{4}\left[\rho_{\mathrm{d} 0}+A \ln \left(\frac{a}{a_{0}}\right)\right]\right\} \Psi(a)=0 .
$$

After applying a WKB approximation (see Appendix A) and using the change of variable ${ }^{4}$ $u \equiv a / a_{0}$, we get:

$$
\psi_{1}(u) \approx \sqrt{\frac{2}{3 \eta} \frac{1}{u^{2}}}\left[\Omega_{\mathrm{d} 0}+\Omega_{A} \ln (u)\right]^{-\frac{1}{4}}\left\{C_{1} e^{i S_{0}(u)}+C_{2} e^{-i S_{0}(u)}\right\}
$$

where $C_{1}$ and $C_{2}$ are constants and we have gathered all the parameters in $\eta \equiv \pi a_{0}^{3} H_{0} / G \hbar$. On the other hand, $S_{0}(u)$ is a real function which can be approximated as (see Appendix A for detailed calculations)

$$
S_{0}(u) \approx \eta \frac{u^{3}}{2}\left[\Omega_{\mathrm{d} 0}+\Omega_{A} \ln (u)\right]^{\frac{1}{2}} .
$$

As can be seen, the wave function vanishes at large scale factors where the LSBR event takes place. Therefore, we can conclude that within the chosen factor ordering, the DeWitt boundary condition is satisfied.

\subsubsection{Second Factor Ordering}

This quantisation procedure is inspired by the Laplace-Beltrami operator, which is defined by the configuration of the variables in the minisuperspace. In this part of the analysis, the single degree of freedom corresponds to the scale factor. Under this factor ordering, the corresponding quantum operator of the conjugate momenta reads

$$
\frac{p_{a}^{2}}{a} \rightarrow \frac{\hat{p}_{a}^{2}}{a}=-\hbar^{2}\left[a^{-\frac{1}{2}} \frac{d}{d a}\right]\left[a^{-\frac{1}{2}} \frac{d}{d a}\right]=-\frac{9}{4} \frac{\hbar^{2}}{a_{0}^{3}} \frac{d^{2}}{d x^{2}},
$$

where we have applied the change of variable $x=\left(a / a_{0}\right)^{\frac{3}{2}}$ to diagonalise the operator. Therefore, the WDW equation is written as

$$
\left[\frac{d^{2}}{d x^{2}}+\eta^{2} x^{2}\left(\Omega_{\Lambda}+\frac{2}{3} \Omega_{A} \ln (x)\right)\right] \psi(a)=0
$$

3 Please notice that this procedure is well defined for any finite value of $a$ and in addition the resulting WDW Equation (23) has WKB solutions that fulfill the DeWitt condition. Moreover, such an approximation is better the larger is the scale factor Before the quantum regime is reached, one would expect to enter in a semi-classical region. It is in that region that the WDW equation multiplied by the scale factor definitely holds, and as we show below the wave function tends to zero for large values of the scale factor.

4 Notice that, in this case, we regard the scale factor as dimensional quantity, so $a_{0}$ corresponds with the current size of the Universe. This parameter is just necessary for a correct dimensional analysis and is reabsorbed by the constant term $\eta$. 
After applying a WKB approximation, we get (see Appendix A)

$$
\psi(x) \approx \frac{1}{\sqrt{\eta}}\left\{x^{2}\left[\Omega_{\Lambda}+\frac{2}{3} \Omega_{A} \ln (x)\right]\right\}^{-\frac{1}{4}}\left\{\tilde{C_{1}} e^{i Q_{0}(x)}+\tilde{C}_{2} e^{-i Q_{0}(x)}\right\},
$$

where $\tilde{C}_{1}$ and $\tilde{C}_{2}$ are constants and $Q_{0}(x)$ is a positive function which can be approximated as (see Appendix A for detailed calculations)

$$
Q_{0}(x) \approx \eta \frac{x^{2}}{2}\left[\Omega_{\Lambda}+\Omega_{A} \ln (x)\right]^{\frac{1}{2}} .
$$

Once again, the wave function vanishes at large scale factors fulfilling the DeWitt boundary condition.

\subsection{WDW Equation with a Scalar Field}

In this section, we address the WDW equation when the matter content is given by a phantom scalar field. Notice that, within this description, the system has a second degree of freedom. The new variable can be understood as a true dynamical degree of freedom while the scale factor plays the role of time. We consider an isotropic and homogeneous Universe where the geometry is given by a FLRW space-time metric. The gravitational action with a minimally coupled (phantom) scalar field is written as $[40,43]$.

$$
S=\int N\left[\frac{3 \pi}{4 G}\left(-\frac{a \dot{a}^{2}}{N^{2}}+k a\right)-\pi^{2} a^{3}\left(\frac{\dot{\phi}^{2}}{N^{2}}+2 V(\phi)\right)\right] d t .
$$

Therefore, the Lagrangian and Hamiltonian can be written, respectively, as

$$
\begin{aligned}
& L=N\left[\frac{3 \pi}{4 G}\left(-\frac{a \dot{a}^{2}}{N^{2}}+k a\right)-\pi^{2} a^{3}\left(\frac{\dot{\phi}^{2}}{N^{2}}+2 V(\phi)\right)\right], \\
& H=N\left[-\frac{G}{3 \pi} \frac{p_{a}^{2}}{a}-\frac{3 \pi k}{4 G} a-\frac{1}{4 \pi^{2}} \frac{p_{\phi}^{2}}{a^{3}}+2 \pi^{2} a^{3} V(\phi)\right],
\end{aligned}
$$

where $p_{\phi}$ is the conjugate momenta corresponding to the scalar field. For the quantisation procedure we choose the Laplace-Beltrami factor ordering. Therefore, the conjugate momenta now become

$$
\frac{p_{a}^{2}}{a} \rightarrow \frac{\hat{p}_{a}^{2}}{a}=-\hbar^{2} \frac{1}{a^{3}}\left[a \frac{d}{d a}\right]\left[a \frac{d}{d a}\right]=-e^{-3 \alpha} \frac{\hbar^{2}}{a_{0}^{3}} \frac{d^{2}}{d \alpha^{2}}, \quad \frac{p_{\phi}^{2}}{a^{3}} \rightarrow \frac{\hat{p}_{\phi}^{2}}{a^{3}}=-e^{-3 \alpha} \frac{\hbar^{2}}{a_{0}^{3}} \frac{d^{2}}{d \phi^{2}}
$$

where we apply the change $\alpha=\ln \left(a / a_{0}\right)$ to diagonalise the operator. Notice that, as the minisuperspace has two degrees of freedom, the operator over the scale factor is different in comparison with the one given in Equation (26). Finally, replacing the previous operators and simplifying the expression, the WDW equation reads

$$
\frac{\hbar^{2}}{2}\left[\frac{\kappa^{2}}{6} \frac{\partial^{2}}{\partial \alpha^{2}}+\frac{\partial^{2}}{\partial \phi^{2}}\right] \Psi(\alpha, \phi)+a_{0}^{6} e^{6 \alpha} V(\phi) \Psi(\alpha, \phi)=0 .
$$

This second order differential equation is quite complicated to solve and we consider a Born-Oppenheimer (BO) approximation [40]. This method provides an approximated solution to the wave function after imposing some conditions. First, the following ansatz is assumed:

$$
\Psi(\alpha, \phi) \equiv \sum_{k} \Psi_{k}(\alpha, \phi), \quad \text { where } \quad \Psi_{k}(\alpha, \phi) \approx \varphi_{k}(\alpha, \phi) C_{k}(\alpha),
$$

where the total wave function is a sum of the product of gravitational part solution, $C_{k}(x)$, and matter part solution, $\varphi_{k}(\alpha, \phi)$. The BO approximation assumes that the back reaction of matter into the 
gravitational part is negligible. The gravitational part changes more rapidly (with respect to $x$ ) than the matter part does. Therefore, the terms that contain derivatives of $\varphi$ respect to $x$ are disregarded, i.e., $\left(\partial^{2} \varphi / \partial \alpha^{2}\right),(\partial \varphi / \partial \alpha)(\partial C / \partial \alpha) \ll\left(\partial^{2} \varphi / \partial \phi^{2}\right),\left(\partial^{2} C / \partial \alpha^{2}\right)$. Within this approximation the differential Equation (34) splits into the following ones

$$
\begin{gathered}
\frac{\hbar^{2}}{2} \frac{\partial^{2} \varphi_{k}(\alpha, \phi)}{\partial \phi^{2}}+\left\{a_{0}^{6} e^{6 \alpha}\left[\frac{A}{6}+2 \pi G A\left(\phi-\phi_{1}\right)^{2}\right]-E_{k}(\alpha)\right\} \varphi_{k}(\alpha, \phi)=0 \\
\frac{\hbar^{2} \kappa^{2}}{6} \frac{\partial^{2} C_{k}(\alpha)}{\partial \alpha^{2}}+2 E_{k}(\alpha) C_{k}(\alpha)=0
\end{gathered}
$$

where the potential $V(\phi)$ has been replaced according to Equation (15) and $E_{k}(\alpha)$ is the eigenvalue that decouples the gravitational and matter part solutions.

As can be seen, the differential Equation (36) is similar to the harmonic oscillator but with the sign switched. This is a direct consequence of considering a phantom scalar field as the potential term behaves as a repulsor rather than the usual harmonic oscillator. The solution to the matter part differential equation is given by the following linear combination

$$
\varphi_{k}(\alpha, \phi)=c_{1} \varphi_{k}^{(1)}(\alpha, \phi)+c_{2} \varphi_{k}^{(2)}(\alpha, \phi)+c_{3} \varphi_{k}^{(3)}(\alpha, \phi)+c_{4} \varphi_{k}^{(4)}(\alpha, \phi),
$$

where the parameters $c_{i}$ are constants and $\varphi_{k}^{(i)}$ the solutions to the differential equation of the matter part. Since Equation (36) is a second order differential equation, only two of the four $\varphi_{k}^{(i)}$ functions given in Equation (38) are linearly independent. These functions can be written as $[44,45]$

$$
\begin{aligned}
& \varphi_{k}^{(1,2)}(\alpha, \phi)=W(\beta, \pm z) \\
& \varphi_{k}^{(3)}(\alpha, \phi)=K^{-1 / 2} W(\beta, z)+i K^{1 / 2} W(\beta,-z) \\
& \varphi_{k}^{(4)}(\alpha, \phi)=K^{-1 / 2} W(\beta, z)-K^{1 / 2} W(\beta,-z)
\end{aligned}
$$

where $W(\beta, z)$ is the parabolic cylinder function and the functions $K, \beta$ and $z$ are defined as

$$
\begin{aligned}
& K=\sqrt{1+e^{2 \pi \beta}}-e^{\pi \beta}, \\
& \beta=-\frac{1}{2 \hbar(\pi G)^{1 / 2}}\left[\frac{A^{1 / 2} a_{0}^{3} e^{3 \alpha}}{6}-\frac{E_{k}}{A^{1 / 2} a_{0}^{3} e^{3 \alpha}}\right], \\
& z=\frac{2 a_{0}^{3 / 2} e^{3 \alpha / 2}(\pi G A)^{1 / 4}}{\hbar^{1 / 2}}\left(\phi-\phi_{1}\right) .
\end{aligned}
$$

It can be verified that when the LSBR singularity occurs, i.e., $\alpha \rightarrow \infty$, the parabolic cilinder functions decrease, as $W(\beta, z) \sim e^{-3 \alpha / 4} \cos \left(e^{3 \alpha}\right)$ and $W(\beta,-z) \sim e^{-3 \alpha / 4} \sin \left(e^{3 \alpha}\right)$. Therefore, for large values of the scale factor the matter part of the wave function vanishes. However, this is not enough to ensure the compliance of the DeWitt boundary condition. We have to check if the gravitational part is bounded at large scale factors. The solutions of the gravitational part are simply given by exponential functions. In the case of a negative value of the parameter $E_{k}$, the solutions are given by 


$$
C_{k}(x)=b_{1} e^{i \frac{\sqrt{12 E_{k}}}{\kappa \hbar} \alpha}+b_{2} e^{-i \frac{\sqrt{12 E_{k}}}{\kappa \hbar} \alpha},
$$

where $b_{1}$ and $b_{2}$ are constants. The exponentials have an imaginary argument, so the solutions are bounded and the DeWitt boundary condition is satisfied ${ }^{5}$. In the case of a positive value of $E_{k}$, the solutions of the gravitational part read

$$
C_{k}(\alpha)=\tilde{b}_{1} e^{\frac{\sqrt{12\left|E_{k}\right|}}{\kappa \hbar} \alpha}+\tilde{b}_{2} e^{-\frac{\sqrt{12\left|E_{k}\right|}}{\kappa \hbar} \alpha},
$$

where $\tilde{b}_{1}$ and $\tilde{b}_{2}$ are constants. In this case, the argument in the exponential is real. Therefore, we set $\tilde{b}_{1}=0$ to avoid the exponentially increasing solutions, thus the DeWitt boundary condition is satisfied as well in the gravitational sector.

\section{Discussion}

In this work, we have analysed the LSBR abrupt event from both a classical and a quantum point of view. First, we have presented the model that induces the LSBR abrupt event. This model can be understood as a simple deviation from the widely accepted $\Lambda$ CDM paradigm and, therefore, a good candidate to describe the current acceleration of the Universe. The main difference when comparing the model against $\Lambda \mathrm{CDM}$ lies in the equation of state Equation (5), which incorporates an additional constant term proportional to the parameter $\mathcal{A}$. For a positive $\mathcal{A}$, the case in which we are interested, the null energy condition is not preserved, hence the designation of phantom matter.

The value of the constant $\mathcal{A}$ can set to be small enough so that the model at present behaves almost as $\Lambda$ CDM. However, the tiniest deviation has important implications on the evolution of the Universe, in the $\Lambda$ CDM model the asymptotic evolution leads to a de Sitter Universe while in the considered model the Universe evolves towards a LSBR event. We refer to this incident as an abrupt event since it occurs at an infinite cosmic time. In fact, when $t \rightarrow \infty$ and $a \rightarrow \infty$ : The Hubble rate blows up. On the contrary, the cosmic time derivative of the Hubble rate remains constant, in this sense, the LSBR abrupt event has a reduced number of diverging parameters in comparison with other singularities and abrupt events like Big Rip [23], Big Freeze [15] or Little Rip [34].

We have considered as well a dark energy content described by a phantom scalar field. The corresponding potential has a quadratic dependence with the scalar field, as given in Equation (15). We notice that the obtained potential show a lower dependence with respect to the scalar field, $\phi$, when comparing with the resulting potentials of other DE models like those that induce the BR and LR events [46,47]. We could affirm that the stronger is the dependence of the potential $V(\phi)$ with respect to $\phi$, the more relevant are the divergences of the cosmological variables. In this sense, we could claim that the LSBR abrupt event is the smoother cosmological incident between the most known singularities and abrupt events. Despite this, all the bound structures in the Universe will be annihilated no matter the size [37], starting from near galaxies, then, the solar system and finally, the Universe will end up destroying the molecular structures and atoms. The LSBR abrupt event occurs at very high energies, where quantum effects are expected to become important.

We have addressed the quantisation of the model describing the dark energy content first with a perfect fluid, and secondly, with a phantom scalar field. In the first case, we have adopted two quantisation procedures where we have made use of a WKB approximation. In both factor orderings, the obtained wave functions fulfill the DeWitt boundary condition, which a priori indicates that the factor ordering has no influence on the compliance of such condition.

In the second case, we have applied a BO approximation, which lead to the possibility to split the wave function into the gravitational and matter part. The wave function describing the matter part

5 The total wave function is a product of the gravitational and matter part solutions. Therefore, the total output vanishes if the wave function of the matter part vanishes faster than the gravitational part could diverge. 
vanishes at large scale factors while the wave function of the gravitational part is bounded for $E_{k}<0$ and decreases exponentially for $0<E_{k}$. Therefore, we can conclude that the DeWitt condition is also satisfied when a minimally coupled phantom scalar field describes the dark energy content.

In spite of all the evidence, we cannot asseverate that the singularities arisen in the classical theory are avoided. We should take these results as a hint of singularity avoidance rather than a full proof.

Acknowledgments: The work of MBL is supported by the Basque Foundation of Science Ikerbasque. The partial support from the Basque government Grant No. IT956-16 (Spain) and FONDOS FEDER under grant FIS2014-57956-P (Spanish government) is also acknowledged. FC acknowledges financial support of the Fundação para a Ciência e Tecnologia (FCT) through the grant PD/BD/128017/2016 and Programa de Doutoramento FCT, PhD: SPACE Doctoral Network for Space Sciences (PD/00040/2012). PMM acknowledges financial support from the projects FIS2014-52837-P (Spanish MINECO) and FIS2016-78859-P (AEI/FEDER, UE).

Author Contributions: The authors contributed equally to this paper.

Conflicts of Interest: The authors declare no conflict of interest.

\section{Appendix A. The WKB Approximation}

In this appendix, we present a brief explanation about the method of the WKB approximation. Let us start with a second order differential equation of the following form:

$$
\left[\frac{d^{2}}{d y^{2}}+V(y)\right] \Psi(y)=0
$$

According to the usual form of the one dimensional Schrödinger equation, the term $V(y)$ can be interpreted as an effective potential. The first order WKB approximation gives the following approximated wave function $\Psi[48]$

$$
\Psi(y) \approx[-V(y)]^{-\frac{1}{4}}\left[B_{1} e^{i S_{0}(y)}+B_{2} e^{-i S_{0}(y)}\right],
$$

where $B_{1}$ and $B_{2}$ are constants and

$$
S_{0}(y)=\int_{y_{1}}^{y} \sqrt{V(y)} d y .
$$

The value of $y_{1}$ in the former equation is assumed to be large enough in such a way that the integration starts in a fully dark energy dominated Universe. As can be seen, for large values of $y$ the potential $V(y)$ diverges towards positive infinite values and the wave function vanishes. On the other hand, the method is valid as long as the following inequality is satisfied [48]

$$
\left|\frac{5 V^{\prime 2}(y)-4 V^{\prime \prime}(y) g(y)}{16 V^{3}(y)}\right| \ll 1,
$$

where prime stands for derivatives with respect to $y$. In the present work, we have applied the WKB approximation to both differential equations obtained after performing different factor orderings. The resulting effective potentials can be generalised with a single expression as

$$
V(y) \equiv \tilde{\eta}^{2} y^{2 s}\left[\Omega_{\mathrm{d} 0}+b \ln (y)\right],
$$

where parameters $s, b$ and $\tilde{\eta}$ take the following values depending on the chosen factor ordering:

$$
\begin{array}{ll}
s=2, \quad b=\Omega_{\mathcal{A}}, \tilde{\eta}=\frac{3 \pi a_{0}^{3} H_{0}}{2 G \hbar}, \quad \text { for the first quantisation procedure, } \\
s=1, \quad b=\frac{2}{3} \Omega_{\mathcal{A}}, \tilde{\eta}=\frac{\pi a_{0}^{3} H_{0}}{G \hbar}, \quad \text { for the second quantisation procedure. }
\end{array}
$$


Solving the integral Equation (A3) for an effective potential of the form Equation (A5), we find

$$
S_{0}(y)=\tilde{\eta} \frac{y^{s+1}}{s+1}\left[\Omega_{\mathrm{d} 0}+b \ln (y)\right]^{\frac{1}{2}}-\left.\tilde{\eta} \frac{\sqrt{\pi b} e^{-\frac{\Omega_{\mathrm{d} 0}}{b}(s+1)}}{2(s+1)^{\frac{3}{2}}}\left\{\operatorname{erfi}\left[\left(\frac{s+1}{b}\right)^{\frac{1}{2}} \sqrt{\Omega_{\mathrm{d} 0}+b \ln (y)}\right]\right\}\right|_{y_{1}} ^{y} .
$$

Therefore, the asymptotic evolution can be approximated as

$$
S_{0}(y) \approx \tilde{\eta} \frac{y^{s+1}}{s+1}\left[\Omega_{\mathrm{d} 0}+b \ln (y)\right]^{\frac{1}{2}},
$$

leading to the approximate solutions Equations (25) and (29). On the other hand, considering the generalised effective potential Equation (A5), the inequality that justifies the WKB approximation given in Equation (A4), can be written now as

$$
\frac{1}{\tilde{\eta}^{2}}\left|\frac{y^{-2(s+1)}}{\left.\mid \Omega_{\mathrm{d} 0}+b \ln (y)\right]^{2}}\right|\left|\frac{5\left\{b+2 s\left[\Omega_{\mathrm{d} 0}+b \ln (y)\right]\right\}^{2}}{16\left[\Omega_{\mathrm{d} 0}+b \ln (y)\right]}-\frac{1}{4}\left\{(2 s-1)\left\{b+2 s\left[\Omega_{\mathrm{d} 0}+b \ln (y)\right]\right\}+2 s b\right\}\right| \ll 1 .
$$

Therefore, the WKB approximation is valid when $y \rightarrow \infty$ since in both quantisation methods $-1<s$.

\section{References}

1. Riess, A.G.; Filippenko, A.V.; Challis, P.; Clocchiatti, A.; Diercks, A.; Garnavich, P.M.; Gilliland, R.L.; Hogan, C.J.; Jha, S.; Supernova Search Team Collaboration; et al. Observational evidence from supernovae for an accelerating universe and a cosmological constant. Astron. J. 1998, 116, 1009.

2. Perlmutter, S.; Aldering, G.; Goldhaber, G.; Knop, R.A.; Nugent, P.; Castro, P.G.; Deustua, S.; Fabbro, S.; Goobar, A.; Supernova Cosmology Project Collaboration; et al. Measurements of Omega and Lambda from 42 high redshift supernovae. Astrophys. J. 1999, 517, 565-585.

3. Ade, P.A.R.; Bock, J.J.; Crill, B.P.; Doré, O.; Helou, G.; Hildebrandt, S.R.; Pearson, T.J.; Prézeau, G.; Seiffert, M.D.; Planck Collaboration; et al. Planck 2015 results. XIV. Dark energy and modified gravity. Astron. Astrophys. 2016, 594, A14.

4. Ade, P.A.R.; Planck Collaboration; Aghanim, N.; Arnaud, M.; Ashdown, M.; Aumont, J.; Baccigalupi, C.; Banday, A.; Barreiro, R.; Bartlett, J.; et al. Planck 2015 results. XIII. Cosmological parameters. Astron. Astrophys. 2016, 594, A13.

5. Tsujikawa, S. Dark energy: Investigation and modeling. Physics 2010, 370, 331-402.

6. Bamba, K.; Capozziello, S.; Nojiri, S.; Odintsov, S.D. Dark energy cosmology: The equivalent description via different theoretical models and cosmography tests. Astrophys. Space Sci. 2012, 342, 155-228.

7. Amendola, L.; Tsujikawa, S. Dark Energy: Theory and Observations, 1st ed.; Cambridge University Press: Cambridge, UK, 2010.

8. Hawking, S.W.; Penrose, R. The Singularities of gravitational collapse and cosmology. Proc. Roy. Soc. Lond. A 1970, 314, 529-548.

9. Hawking, S.W.; Penrose, R. The Nature of Space and Time; Princeton University Press: Princeton, NJ, USA, 1996.

10. Barrow, J.D.; Galloway, G.J.; Tipler, F.J. The closed-universe recollapse conjecture. Mon. Not. R. Astron. Soc. 1986, 223, 835-844.

11. Barrow, J.D. Sudden future singularities. Class. Quantum Gravity 2004, 21, L79-L82.

12. Gorini, V.; Kamenshchik, A.; Moschella, U.; Pasquier, V. The Chaplygin gas as a model for dark energy. Phys. Rev. D 2004, 69, 123512.

13. Nojiri, S.; Odintsov, S.D.; Tsujikawa, S. Properties of singularities in (phantom) dark energy universe. Phys. Rev. D 2005, 71, 063004.

14. Barrow, J.D.; Tsagas, C.G. New isotropic and anisotropic sudden singularities. Class. Quantum Gravity 2005, $22,1563$. 
15. Bouhmadi-López, M.; González-Díaz, P.F.; Martín-Moruno, P. On the generalised Chaplygin gas: Worse than a big rip or quieter than a sudden singularity? Int. J. Mod. Phys. D 2008, 17, 2269-2290.

16. Bouhmadi-López, M.; González-Díaz, P.F.; Martín-Moruno, P. Worse than a big rip? Phys. Lett. B 2008, 659, $1-5$.

17. Nojiri, S.; Odintsov, S.D. The Final state and thermodynamics of dark energy universe. Phys. Rev. D 2004, 70, 103522.

18. Nojiri, S.; Odintsov, S.D. Inhomogeneous equation of state of the universe: Phantom era, future singularity and crossing the phantom barrier. Phys. Rev. D 2005, 72, 023003.

19. Bamba, K.; Nojiri, S.; Odintsov, S.D. The Universe future in modified gravity theories: Approaching the finite-time future singularity. J. Cosmol. Astropart. Phys. 2008, 810, 045.

20. Nojiri, S.; Odintsov, S.D. The Future evolution and finite-time singularities in $\mathrm{F}(\mathrm{R})$-gravity unifying the inflation and cosmic acceleration. Phys. Rev. D 2008, 78, 046006.

21. Bouhmadi-López, M.; Kiefer, C.; Krämer, M. Resolution of type IV singularities in quantum cosmology. Phys. Rev. D 2014, 89, 064016.

22. Nojiri, S.; Odintsov, S.D.; Oikonomou, V.K.; Saridakis, E.N. Singular cosmological evolution using canonical and ghost scalar fields. J. Cosmol. Astropart. Phys. 2015, 1509, 044.

23. Starobinsky, A.A. Future and origin of our universe: Modern view. Gravit. Cosmol. 2000, 6, 71-84.

24. Caldwell, R.R. A Phantom menace? Phys. Lett. B 2002, 545, 23.

25. Caldwell, R.R.; Kamionkowski, M.; Weinberg, N.N. Phantom energy and cosmic doomsday. Phys. Rev. Lett. 2003, 91, 071301.

26. Carroll, S.M.; Hoffman, M.; Trodden, M. Can the dark energy equation-of-state parameter w be less than -1 ? Phys. Rev. D 2003, 68, 1557-1567.

27. Chimento, L.P.; Lazkoz, R. On the link between phantom and standard cosmologies. Phys. Rev. Lett. 2003, 91, 211301.

28. Dąbrowski, M.P.; Stachowiak, T.; Szydlowski, M. Phantom cosmologies. Phys. Rev. D 2003, 68, 103519.

29. González-Díaz, P.F. K-essential phantom energy: Doomsday around the corner? Phys. Lett. B 2004, 586, 1-4.

30. González-Díaz, P.F. Axion phantom energy. Phys. Rev. D 2004, 69, 063522.

31. Ruzmaikina, T.; Ruzmaikin, A.A. Quadratic corrections to the Lagrangian density of the gravitational field and the singularity. Sov. Phys. JETP 1970, 30, 372.

32. Štefančić, H. Expansion around the vacuum equation of state-Sudden future singularities and asymptotic behavior. Phys. Rev. D 2005, 71, 084024.

33. Bouhmadi-López, M. Phantom-like behaviour in dilatonic brane-world scenario with induced gravity. Nucl. Phys. B 2008, 797, 78-92.

34. Frampton, P.H.; Ludwick, K.J.; Scherrer, R.J. The Little Rip. Phys. Rev. D 2011, 84, 063003.

35. Brevik, I.; Elizalde, E.; Nojiri, S.; Odintsov, S.D. Viscous Little Rip Cosmology. Phys. Rev. D 2011, 84, 103508.

36. Bouhmadi-López, M.; Chen, P.; Liu, Y.-W. Tradeoff between smoother and sooner 'little rip'. Eur. Phys. J. C 2013, 73, 2546.

37. Bouhmadi-López, M.; Errahmani, A.; Martín-Moruno, P.; Ouali, T.; Tavakoli, Y. The little sibling of the big rip singularity. Int. J. Mod. Phys. D 2015, 24, 1550078.

38. Morais, J.; Bouhmadi-López, M.; Kumar, K.S.; Marto, J.; Tavakoli, Y. Interacting 3-form dark energy models: Distinguishing interactions and avoiding the Little Sibling of the Big Rip. Phys. Dark Universe 2017, 15, 7-30.

39. Ita, E.E., III; Soo, C.; Yu, H.L. Intrinsic Time Quantum Geometrodynamics. Prog. Theor. Exp. Phys. 2015, 2015, 083E01.

40. Kiefer, C. Quantum Gravity, 2nd ed.; Oxford University Press: Oxford, UK, 2007.

41. Moniz, P. Quantum Cosmology -The Supersymmetric Perspective-Vol.1: Fundamentals; Lecture Notes in Physics; 803; Springer: Berlin/Heidelberg, Germany, 2010.

42. DeWitt, B.S. Quantum Theory of Gravity. 1. The Canonical Theory. Phys. Rev. 1967, 160, 1113-1148.

43. Dąbrowski, M.P.; Kiefer, C.; Sandhöfer, B. Quantum phantom cosmology. Phys. Rev. D 2006, 74, 044022.

44. Albarran, I.; Bouhmadi-López, M.; Cabral, F.; Martín-Moruno, P. The quantum realm of the "Little Sibling" of the Big Rip singularity. J. Cosmol. Astropart. Phys. 2015, 1511, 044.

45. Abramowitz, M.; Stegun, I. Handbook on Mathematical Functions; Dover Publications: Dover, UK, 1980.

46. Hao, J.G.; Li, X.Z. An Attractor solution of phantom field. Phys. Rev. D 2003, 67, 107303. 
47. Albarran, I.; Bouhmadi-López, M.; Kiefer, C.; Marto, J.; Moniz, P.V. Classical and quantum cosmology of the little rip abrupt event. Phys. Rev. D 2016, 94, 063536.

48. Mathews, J.; Walker, R.L. Mathematical Methods of Physics; California Institute of Techonolgy: Pasadena, CA, USA, 1969.

(C) 2018 by the authors. Licensee MDPI, Basel, Switzerland. This article is an open access article distributed under the terms and conditions of the Creative Commons Attribution (CC BY) license (http://creativecommons.org/licenses/by/4.0/). 